\title{
PRIORITIZATION OF ADMINISTRATIONS TO CONNECT TO THE INTERNAL NETWORK WITH A MULTIPLE CRITERIA DECISION MAKING APPROACH

\author{
(CASE STUDY: INFORMATION TECHNOLOGY ADMINISTRATION OF \\ IRAN)
}

\author{
Somayeh Movahedy \\ Department of Communication Science, Tehran Shargh Branch, Islamic Azad University, Tehran, Iran \\ M.A Student at Firoozkooh Azad University, and a Master of Tax \\ mahdi.mahdinia@yahoo.com
}

\begin{abstract}
The internal network consists of communication substructures, governmental and nongovernmental developed data centers and software substructures that have been expanded across the nation. This network provides the required capacity for "maintenance and exchange of local information in the nation for electronic services development" and "access of internet" through nationwide bandwidth connecting bed for home users, businesses and executive administrations. This network integrates specialized, local and national networks in the country, consisting of two private and public sectors. The private sector is to connect and to exchange information and services between the executive administrations and private sector, providing the required services for general users. These two sectors join each other at such points as data centers. The problem of this research is the fact that since many national administrations have a number of access points and that all administrations must join this network within the next five years, and that identifying information technology indices such as existing points, connected to national headquarters and number of existing points, connected to provincial and city offices and number of existing and connected points of associated businesses for executive administrations of the nation is of great importance for materialization of an electronic government in the nation, in this research, we have been determined to prioritize the aforesaid factors and indices. The research method is of descriptive mathematical type and the technique used is TOPSIS. Moreover, the names of ministries have not been given herein this survey.
\end{abstract}

Keywords: National Information Network, TOPSIS and Connection Points

\section{INTRODUCTION}

Information technology is more extensive (and complicated) than computer science. This term replaced the terms of data processing and management information systems in the $1990 \mathrm{~s}$, which was very common in the 19760s and 1970s. Information technology usually implies all technologies that are applied in five areas of collection, saving, processing, transfer and display of data. Information technology knowledge and computer are different from each other. They have many items in common. If computer science is considered similar to mechanical engineering, information technology is like transportation. In transportation industry, automobiles, railways, airplanes and ships are available. All of these items are put forth by mechanical engineers. Meanwhile, in transportation industry, the issues associated with navigation management, traffic management, determination of transportation strategy at the levels of company, city and country. It does not directly correspond to mechanical engineering. However, information technology and communication (ICT), which is said as FAVA in Persian, is the most important notation in this field. Seyed Hamed Khosravani Shariati propounds another definition of information technology. As he says, information technology engineering is a combined knowledge of software engineering, industrial engineering and marketing with an analytical, commercial and narrow-sighted 
approach toward modern information technologies. Having put forth this approach, he solved the gap between software designers, system analysts and target market. The problem of this research is the fact that in spite of many access points by national administrations and that all administrations must connect to this network, on the one hand and on the other hand, since identifying IT indices such as number of existing and connected points of the national headquarters, number of existing and connected points of provincial and city offices and number of existing and connected points of affiliated business is of great importance for realization of an electronic government, herein this research, we have been determined to prioritize state administrations in terms of the aforesaid elements and indices. The technique used for prioritization is multiple decision making and TOPSIS in particular.

\section{INTERNAL NETWORK}

An internal network consists of communication substructures, advanced governmental and nongovernmental data centers and software substructures that have been extended across the nation. This network provides the required capacity for "maintenance and safe exchange of local information in the nation for development of electronic services" and "access of internet" through nationwide bandwidth connection bed for home users, businesses and executive administrations. This network integrates specialized, local and national networks across the nation. It consists of two private and public sectors. The private sector is for communication and exchange of information and services of executive administrations and the public sector is for providing services for general users. These two sectors join each other at such points as data centers(sohrabi et al, 2015).

\section{SHARE CENTER AND EXCHANGE OF INFORMATION}

Share center and exchange of information among governmental administrations are commissioned as subdivision of this network. This center shall be launched upon approval and notification of the by-law for connection of executive administration and share of information among the said administrations. This center is similar to interbank acceleration network and is generated to share information among different administrations.

\section{WHY INTERNAL NETWORK?}

Generation and local development as a legal duty shall provide suitable substructure and required capacity for development of various applications of information technology in the nation. From among the characteristics of this network, one may point out provision of high speed accesses for all users. It consists of data centers as host of data of different types of application. In fact, internal network has notified all legal items for generating a uniform window, information systems and databases as an assigned duty to corresponding administrations and shall support them as substructure. 
ANALYTICAL RESEARCH MODEL

Any field research requires a model indicating the variables and their interrelations in form of an appropriate analytical means.
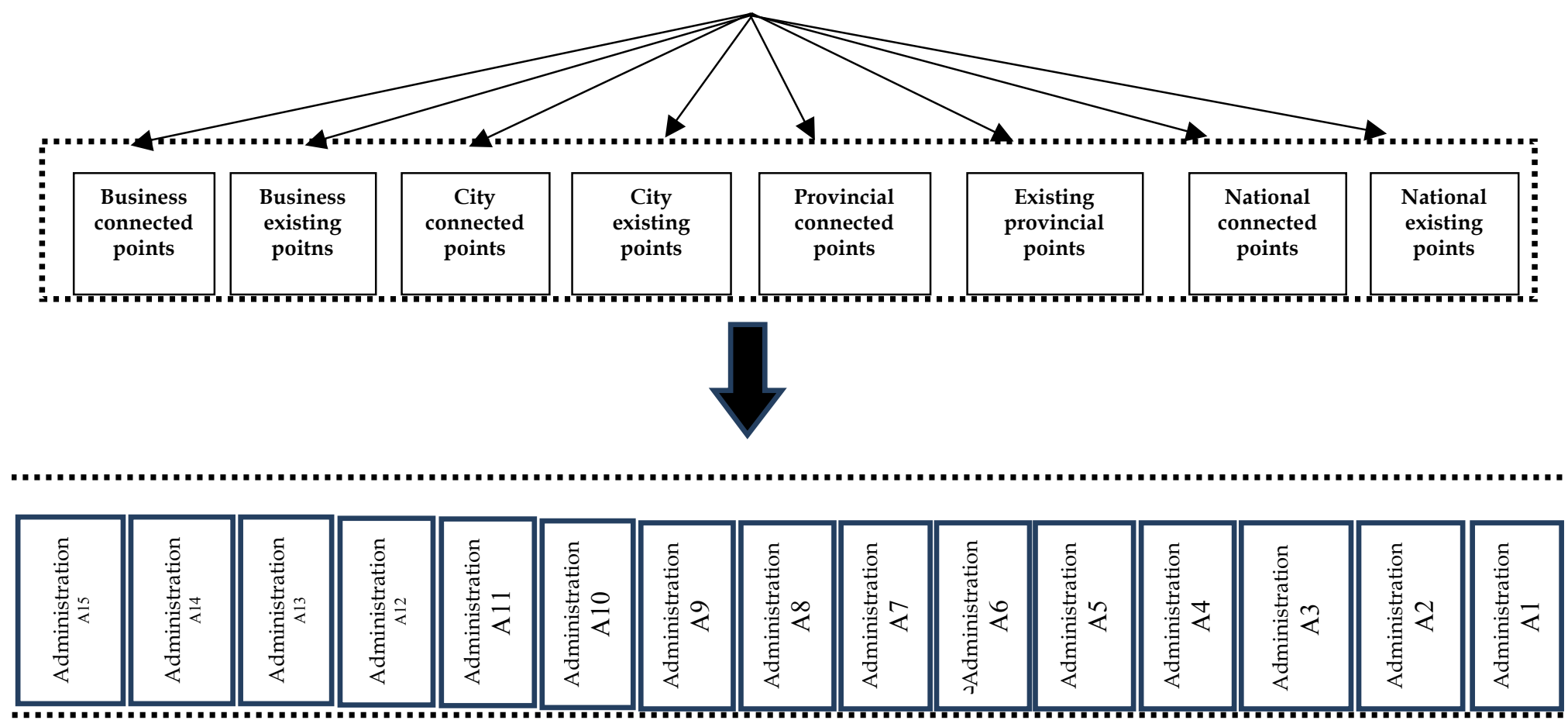

Figure: The conceptual research model based on prioritization of connection of administrations to an internal network

\section{TOPSIS METHOD}

Now, in order to achieve the final rating, these different ratings are put in matrix and will be solved using figures and final rating of executive administrations shall be achieved.

Calculations

Furthermore, decision making and calculations matrixes performed on these matrixes shall be given.

Table 1: Matrix of Decision Making

First, table of decision making (number of connected and existing points of central administrations of the nation with subunits) shall be drawn.

Table 1: Matrix of Decision Making

\begin{tabular}{|c|c|c|c|c|c|c|c|}
\hline Index & $\begin{array}{c}\text { National } \\
\text { Existing } \\
\text { points }\end{array}$ & $\begin{array}{c}\text { National } \\
\text { connected } \\
\text { points }\end{array}$ & $\begin{array}{c}\text { Provincial } \\
\text { existing } \\
\text { points }\end{array}$ & $\begin{array}{c}\text { Provincial } \\
\text { connected } \\
\text { points }\end{array}$ & $\begin{array}{c}\text { city } \\
\text { existing } \\
\text { points }\end{array}$ & $\begin{array}{c}\text { City } \\
\text { connected } \\
\text { points }\end{array}$ & $\begin{array}{c}\text { business } \\
\text { existing } \\
\text { points }\end{array}$ \\
$\begin{array}{c}\text { ponnecting } \\
\text { points }\end{array}$ \\
\hline
\end{tabular}




\begin{tabular}{|c|c|c|c|c|c|c|c|c|}
\hline Administration A1 & 13 & 13 & 64 & 64 & 714 & 714 & 89309 & 39845 \\
\hline Administration A2 & 84 & 81 & 131 & 69 & 0 & 0 & 78 & 78 \\
\hline Administration A3 & 7 & 0 & 30 & 30 & 0 & 0 & 9588 & 0 \\
\hline Administration A4 & 14 & 14 & 49 & 49 & 0 & 0 & 27000 & 9000 \\
\hline Administration A5 & 5 & 5 & 31 & 6 & 380 & 0 & 801 & 0 \\
\hline Administration A6 & 4 & 4 & 257 & 64 & 1408 & 214 & 1483 & 0 \\
\hline Administration A7 & 15 & 15 & 0 & 0 & 1107 & 666 & 0 & 0 \\
\hline Administration A8 & 5 & 5 & 31 & 31 & 1378 & 1378 & 0 & 0 \\
\hline Administration A9 & 5 & 5 & 0 & 0 & 0 & 0 & 911 & 2 \\
\hline Administration A10 & 26 & 12 & 31 & 0 & 93 & 0 & 0 & 0 \\
\hline Administration A11 & 30 & 16 & 31 & 30 & 337 & 291 & 100000 & 10000 \\
\hline Administration A12 & 29 & 24 & 290 & 252 & 1336 & 1050 & 18 & 0 \\
\hline Administration A13 & 12 & 11 & 89 & 79 & 1600 & 1354 & 941 & 904 \\
\hline Administration A14 & 1 & 0 & 31 & 0 & 361 & 0 & 20338 & 0 \\
\hline Administration A 15 & 13 & 13 & 288 & 288 & 9244 & 9244 & 11578 & 11578 \\
\hline Total & 263 & 218 & 1353 & 962 & 17958 & 14911 & 262045 & 71407 \\
\hline
\end{tabular}

\section{TABLE OF WEIGHT OBTAINED FROM EACH ELITE FOR EIGHT CRITERIA}

After we have obtained the importance of all eight criteria from eight elite, we develop table of weights of criteria. Thus, the surface of this table comprises elites and its column indicates existing criteria. At the end, using the average of weights obtained from each criterion, the weight of corresponding weight shall be achieved.

Table 2: The weights obtained from each elite for eight existing criteria

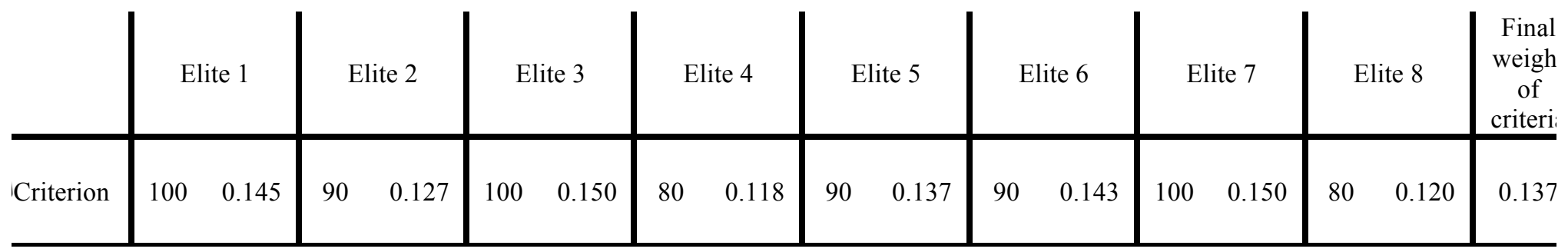


The Turkish Online Journal of Design, Art and Communication - TOJDAC August 2016 Special Edition

\begin{tabular}{|c|c|c|c|c|c|c|c|c|c|c|c|c|c|c|c|c|c|}
\hline riterion 2 & 70 & 0.102 & 100 & 0.217 & 90 & 0.135 & 70 & 0.103 & 80 & 0.122 & 80 & 0.127 & 90 & 0.135 & 70 & 0.105 & 0.131 \\
\hline Yriterion 3 & 90 & 0.130 & 80 & 0.140 & 100 & 0.150 & 100 & 0.147 & 60 & 0.090 & 100 & 0.159 & 80 & 0.120 & 90 & 0.135 & 0.134 \\
\hline riterion 4 & 100 & 0.145 & 70 & 0.098 & 90 & 0.135 & 90 & 0.133 & 50 & 0.076 & 80 & 0.127 & 90 & 0.135 & 60 & 0.090 & 0.118 \\
\hline Yriterion 5 & 100 & 0.145 & 100 & 0.217 & 80 & 0.120 & 90 & 0.133 & 100 & 0.152 & 70 & 0.112 & 90 & 0.135 & 100 & 0.150 & 0.146 \\
\hline Yriterion 6 & 60 & 0.087 & 100 & 0.217 & 80 & 0.120 & 80 & 0.118 & 90 & 0.137 & 80 & 0.127 & 70 & 0.105 & 90 & 0.135 & 0.131 \\
\hline riterion 7 & 90 & 0.130 & 90 & 0.127 & 60 & 0.090 & 100 & 0.147 & 90 & 0.137 & 70 & 0.112 & 80 & 0.120 & 80 & 0.120 & 0.123 \\
\hline riterion 8 & 80 & 0.163 & 80 & 0.140 & 70 & 0.105 & 70 & 0.103 & 100 & 0.152 & 60 & 0.096 & 70 & 0.105 & 100 & 0.150 & 0.127 \\
\hline Total & 690 & 1 & 710 & 1 & 670 & 1 & 680 & 1 & 660 & 1 & 630 & 1 & 670 & 1 & 670 & 1 & 1 \\
\hline
\end{tabular}

Descaling of the figures given in the table:

Since number of existing and connected points of central administrations in order of subdivisions is different in each criterion, we should descale the figures inside each column. For this purpose, we use soft Euclidean formulation.

Table 3: Descaling of the points of decision making matrix

\begin{tabular}{|c|c|c|c|c|c|c|c|c|}
\hline Index & $\begin{array}{c}\text { National } \\
\text { existing } \\
\text { points }\end{array}$ & $\begin{array}{c}\text { National } \\
\text { connected } \\
\text { points }\end{array}$ & $\begin{array}{c}\text { Provincial } \\
\text { existing } \\
\text { points }\end{array}$ & $\begin{array}{c}\text { Provincial } \\
\text { connected } \\
\text { points }\end{array}$ & $\begin{array}{c}\text { City } \\
\text { existing } \\
\text { points }\end{array}$ & $\begin{array}{c}\text { City } \\
\text { connected } \\
\text { points }\end{array}$ & $\begin{array}{c}\text { Existing } \\
\text { business } \\
\text { points }\end{array}$ & $\begin{array}{c}\text { Business } \\
\text { connected } \\
\text { points }\end{array}$ \\
\hline Administration A1 & 0.80 & 0.88 & 1.74 & 2.06 & 5.33 & 5.85 & 174.46 & 149.11 \\
\hline Administration A2 & 5.18 & 5.49 & 3.56 & 2.22 & 0.00 & 0.00 & 0.15 & 0.29 \\
\hline Administration A3 & 0.43 & 0.00 & 0.82 & 0.97 & 0.00 & 0.00 & 18.73 & 0.00 \\
\hline Administration A4 & 0.86 & 0.95 & 1.33 & 1.58 & 0.00 & 0.00 & 52.74 & 33.68 \\
\hline Administration A5 & 0.31 & 0.34 & 0.84 & 0.19 & 2.84 & 0.00 & 1.56 & 0.00 \\
\hline Administration A6 & 0.25 & 0.27 & 6.99 & 2.06 & 10.51 & 1.75 & 2.90 & 0.00 \\
\hline
\end{tabular}




\begin{tabular}{|c|c|c|c|c|c|c|c|c|}
\hline Administration A7 & 0.92 & 1.02 & 0.00 & 0.00 & 8.26 & 5.45 & 0.00 & 0.00 \\
\hline Administration A8 & 0.31 & 0.34 & 0.84 & 1.00 & 10.28 & 11.28 & 0.00 \\
\hline Administration A9 & 0.31 & 0.34 & 0.00 & 0.00 & 0.00 & 0.00 & 1.78 \\
\hline $\begin{array}{c}\text { Administration } \\
\text { A10 }\end{array}$ & 1.60 & 0.81 & 0.84 & 0.00 & 0.69 & 0.00 & 0.00 \\
\hline $\begin{array}{c}\text { Administration } \\
\text { A11 }\end{array}$ & 1.85 & 1.08 & 0.84 & 0.97 & 2.51 & 2.38 & 195.35 \\
\hline $\begin{array}{c}\text { Administration } \\
\text { A12 }\end{array}$ & 1.79 & 1.63 & 7.88 & 8.12 & 9.97 & 8.60 & 0.04 \\
\hline $\begin{array}{c}\text { Administration } \\
\text { A13 }\end{array}$ & 0.74 & 0.75 & 2.42 & 2.55 & 11.94 & 11.09 & 1.84 \\
\hline $\begin{array}{c}\text { Administration } \\
\text { A14 }\end{array}$ & 0.06 & 0.00 & 0.84 & 0.00 & 2.69 & 0.00 \\
\hline $\begin{array}{c}\text { Administration } \\
\text { A15 }\end{array}$ & 0.80 & 0.88 & 7.83 & 9.29 & 68.98 & 75.70 & 22.62 \\
\hline Formation
\end{tabular}

Formation of normalized matrix of descaled weights

For normalization of weights, first, we should write down the weight of each criterion on top of each column.

Table 4: Table of multiplication of weight of each criterion by the column of the same criterion

\begin{tabular}{|c|c|c|c|c|c|c|c|c|}
\hline $\begin{array}{c}\text { The weights of } \\
\text { criteria }\end{array}$ & 0.137 & 0.131 & 0.134 & 0.118 & 0.146 & 0.131 & 0.123 & 0.127 \\
\hline Index & $\begin{array}{l}\text { National } \\
\text { existing } \\
\text { points }\end{array}$ & $\begin{array}{l}\text { National } \\
\text { connected } \\
\text { points }\end{array}$ & $\begin{array}{l}\text { Provincial } \\
\text { existing } \\
\text { points }\end{array}$ & $\begin{array}{l}\text { Provincial } \\
\text { connected } \\
\text { points }\end{array}$ & $\begin{array}{l}\text { City } \\
\text { existing } \\
\text { points }\end{array}$ & $\begin{array}{c}\text { City } \\
\text { connected } \\
\text { points }\end{array}$ & $\begin{array}{l}\text { Business } \\
\text { existing } \\
\text { points }\end{array}$ & $\begin{array}{l}\text { Business } \\
\text { connected } \\
\text { points }\end{array}$ \\
\hline $\begin{array}{c}\text { Administration } \\
\text { A1 }\end{array}$ & 0.80 & 0.88 & 1.74 & 2.06 & 5.33 & 5.85 & 174.46 & 149.11 \\
\hline $\begin{array}{l}\text { Administration } \\
\text { A2 }\end{array}$ & 5.18 & 5.49 & 3.56 & 2.22 & 0.00 & 0.00 & 0.15 & 0.29 \\
\hline $\begin{array}{c}\text { Administration } \\
\text { A3 }\end{array}$ & 0.43 & 0.00 & 0.82 & 0.97 & 0.00 & 0.00 & 18.73 & 0.00 \\
\hline $\begin{array}{c}\text { Administration } \\
\text { A4 }\end{array}$ & 0.86 & 0.95 & 1.33 & 1.58 & 0.00 & 0.00 & 52.74 & 33.68 \\
\hline $\begin{array}{c}\text { Administration } \\
\text { A5 }\end{array}$ & 0.31 & 0.34 & 0.84 & 0.19 & 2.84 & 0.00 & 1.56 & 0.00 \\
\hline $\begin{array}{c}\text { Administration } \\
\text { A6 }\end{array}$ & 0.25 & 0.27 & 6.99 & 2.06 & 10.51 & 1.75 & 2.90 & 0.00 \\
\hline $\begin{array}{c}\text { Administration } \\
\text { A7 }\end{array}$ & 0.92 & 1.02 & 0.00 & 0.00 & 8.26 & 5.45 & 0.00 & 0.00 \\
\hline $\begin{array}{c}\text { Administration } \\
\text { A8 }\end{array}$ & 0.31 & 0.34 & 0.84 & 1.00 & 10.28 & 11.28 & 0.00 & 0.00 \\
\hline $\begin{array}{c}\text { Administration } \\
\text { A9 }\end{array}$ & 0.31 & 0.34 & 0.00 & 0.00 & 0.00 & 0.00 & 1.78 & 0.01 \\
\hline $\begin{array}{l}\text { Administration } \\
\text { A10 }\end{array}$ & 1.60 & 0.81 & 0.84 & 0.00 & 0.69 & 0.00 & 0.00 & 0.00 \\
\hline
\end{tabular}




\begin{tabular}{|c|c|c|c|c|c|c|c|c|}
\hline $\begin{array}{c}\text { Administration } \\
\text { A11 }\end{array}$ & 1.85 & 1.08 & 0.84 & 0.97 & 2.51 & 2.38 & 195.35 & 37.42 \\
\hline $\begin{array}{c}\text { Administration } \\
\text { A12 }\end{array}$ & 1.79 & 1.63 & 7.88 & 8.12 & 9.97 & 8.60 & 0.04 & 0.00 \\
\hline $\begin{array}{c}\text { Administration } \\
\text { A13 }\end{array}$ & 0.74 & 0.75 & 2.42 & 2.55 & 11.94 & 11.09 & 1.84 & 3.38 \\
\hline $\begin{array}{c}\text { Administration } \\
\text { A14 }\end{array}$ & 0.06 & 0.00 & 0.84 & 0.00 & 2.69 & 0.00 & 39.73 & 0.00 \\
\hline $\begin{array}{c}\text { Administration } \\
\text { A15 }\end{array}$ & 0.80 & 0.88 & 7.83 & 9.29 & 68.98 & 75.70 & 22.62 \\
\hline
\end{tabular}

Then, the weight of each criterion is written in all figures of corresponding column to the said criterion and develop the following table, which is normalized table of weights:

Table 5: Normalized Table of Weights of each Criterion

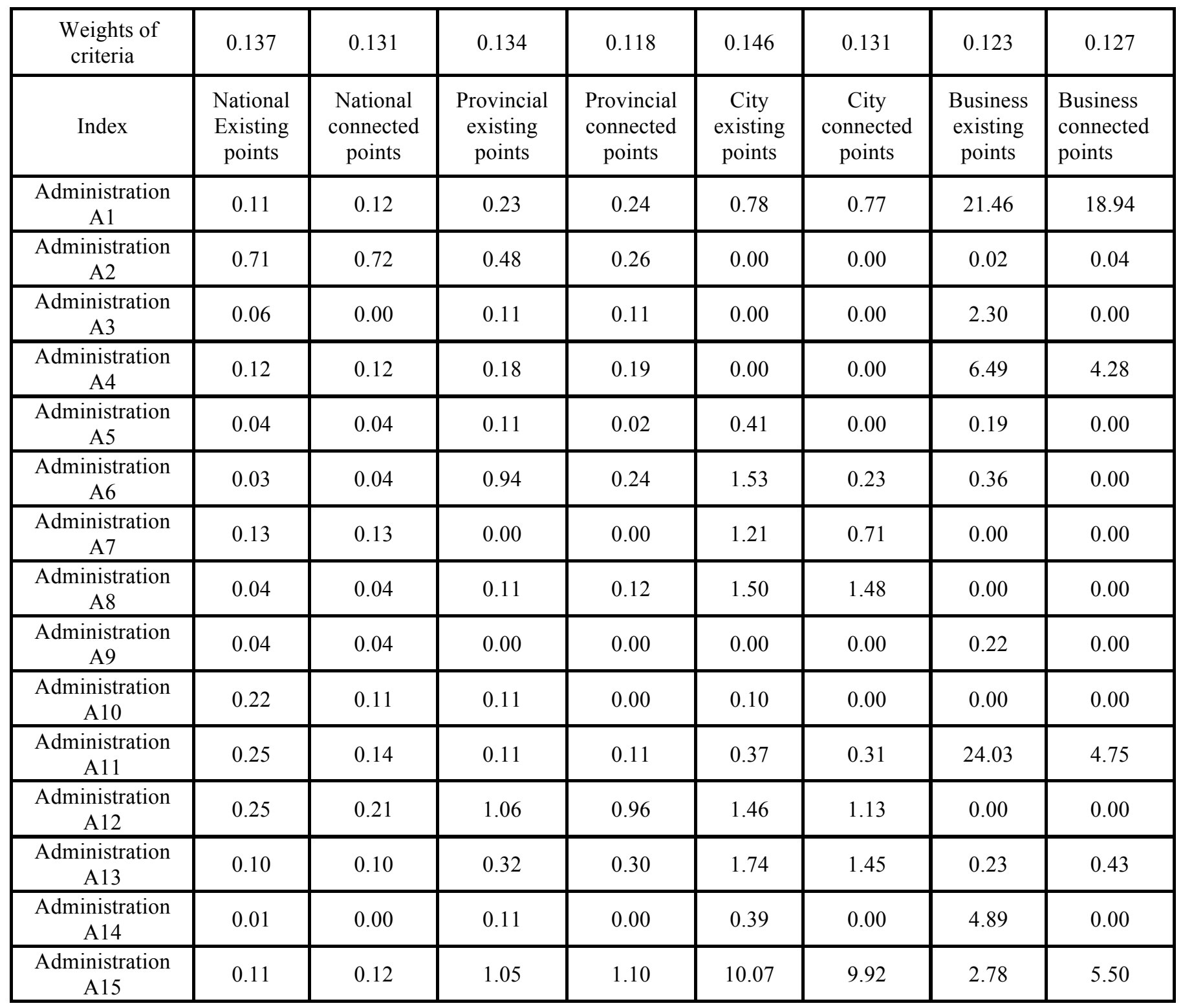


Identifying a ideal positivesolution and a ideal negative solution

Now, we develop the table of positive and ideal negative solutions. For this purpose, we consider the corresponding criteria to existing points of administrations as negative nature (since it has cost nature), and the corresponding criteria to connected points as positive nature. Then, we develop the following table through ideal positive and ideal negative formula.

Table 6: Ideal positive and ideal negative solution

\begin{tabular}{|c|c|c|c|c|c|c|c|c|}
\hline & $\begin{array}{c}\text { National } \\
\text { existing } \\
\text { points }\end{array}$ & $\begin{array}{c}\text { National } \\
\text { connected } \\
\text { points }\end{array}$ & $\begin{array}{c}\text { Provincial } \\
\text { existing } \\
\text { points }\end{array}$ & $\begin{array}{c}\text { Provincial } \\
\text { connected } \\
\text { points }\end{array}$ & $\begin{array}{c}\text { City } \\
\text { existing } \\
\text { points }\end{array}$ & $\begin{array}{c}\text { City } \\
\text { connected } \\
\text { points }\end{array}$ & $\begin{array}{c}\text { Business } \\
\text { existing } \\
\text { points }\end{array}$ & $\begin{array}{c}\text { Business } \\
\text { connected } \\
\text { points }\end{array}$ \\
\hline $\mathrm{A}^{+}$ & 0.01 & 0.72 & 0.00 & 1.10 & 0.00 & 9.92 & 0.00 & 18.94 \\
\hline $\mathrm{A}^{-}$ & 0.71 & 0.00 & 1.06 & 0.00 & 1.74 & 0.00 & 24.03 & 0.00 \\
\hline
\end{tabular}

\section{DISTANCE CALCULATION}

Now that we have calculated the figures of the aforesaid table, we must calculate the distance between each option and ideal positive and ideal negative solution according to the formula for measurement of distances. The following results have been obtained:

$$
\begin{aligned}
& \mathrm{d}^{*} \mathrm{~A} 1=23.37 \\
& \mathrm{~d}^{*} \mathrm{~A} 2=21.38 \\
& \mathrm{~d}^{*} \mathrm{~A} 3=21.53 \\
& \mathrm{~d}^{*} \mathrm{~A} 4=18.88 \\
& \mathrm{~d}^{*} \mathrm{~A} 5=21.42 \\
& \mathrm{~d}^{*} \mathrm{~A} 6=21.38 \\
& \mathrm{~d}^{*} \mathrm{~A} 7=21.13 \\
& \mathrm{~d}^{*} \mathrm{~A} 8=20.82 \\
& \mathrm{~d}^{*} \mathrm{~A} 9=21.42 \\
& \mathrm{~d}^{*} \mathrm{~A} 10=21.41 \\
& \mathrm{~d}^{*} \mathrm{~A} 11=29.53 \\
& \mathrm{~d}^{*} \mathrm{~A} 12=20.96 \\
& \mathrm{~d}^{*} \mathrm{~A} 13=20.45
\end{aligned}
$$


$\mathrm{d}^{*} \mathrm{~A} 14=21.97$

$\mathrm{d}^{*} \mathrm{~A} 15=17.06$

$d^{*} A=\left\{d^{*} A 1, d^{*} A 2, d^{*} A 3, d^{*} A 4, d^{*} A 5, d^{*} A 6, d^{*} A 7, d^{*} A 8, d^{*} A 9, d^{*} A 10, d^{*} A 11, d^{*} 12, d^{*} A 13\right.$, $\left.\mathrm{d}^{*} \mathrm{~A} 14, \mathrm{~d}^{*} \mathrm{~A} 15\right\}$

$\mathrm{d}^{*} \mathrm{~A}=\{23.37,21.38,21.53,18.88,21.42,21.38,21.13,20.82,21.42,21.41,29.53,20.96,20.45,21.97$, $17.06\}$

$$
\mathrm{d}-\mathrm{A} 1=19.18
$$

$\mathrm{d}-\mathrm{A} 2=24.09$

$\mathrm{d}-\mathrm{A} 3=21.82$

d-A4 $=18.17$

$\mathrm{d}-\mathrm{A} 5=23.90$

d-A6 $=23.68$

$\mathrm{d}-\mathrm{A} 7=24.08$

$\mathrm{d}-\mathrm{A} 8=24.10$

$\mathrm{d}-\mathrm{A} 9=23.91$

$\mathrm{d}-\mathrm{A} 10=24.11$

$\mathrm{d}-\mathrm{A} 11=5.07$

d-A12 $=24.08$

d-A13 $=23.87$

d-A14 $=19.22$

$\mathrm{d}-\mathrm{A} 15=25.51$

d-A $=\{$ d-A1 , d-A2 , d-A3 , d-A4 , d-A5 , d-A6 , d-A7 , d-A8 , d-A9, d-A10, d-A11 , d-12, d-A13 , d-A14, dA15\}

$d-A=\{19.18,24.09,21.82,18.17,23.90,23.68,24.08,24.10,23.91,24.11,5.07,24.08,23.87,19.22$, $25.51\}$

Relative closeness and calculation of final figures for prioritization of administrations 
Table 7: Final Administrational Figures

\begin{tabular}{|l|l|}
\hline CA1 & 0.451 \\
\hline CA2 & 0.530 \\
\hline CA3 & 0.503 \\
\hline CA4 & 0.490 \\
\hline CA5 & 0.527 \\
\hline CA6 & 0.526 \\
\hline CA7 & 0.533 \\
\hline CA8 & 0.537 \\
\hline CA9 & 0.527 \\
\hline CA10 & 0.530 \\
\hline CA11 & 0.147 \\
\hline CA12 & 0.535 \\
\hline CA13 & 0.539 \\
\hline CA14 & 0.467 \\
\hline CA15 & 0.599 \\
\hline & \\
\hline
\end{tabular}

Final step: Prioritization of Central National Administrations

Finally, sorting out the figures of the above table on ascending-descending basis, we prioritize the central administrations as follows:

Table 8: Prioritization of Central National Administrations

\begin{tabular}{|c|c|}
\hline Priority & Name of Administration \\
\hline 1 & A15 \\
\hline 2 & A13 \\
\hline 3 & A8 \\
\hline 4 & A12 \\
\hline 5 & A7 \\
\hline 6 & A2 \\
\hline 7 & A10 \\
\hline 8 & A5 \\
\hline 9 & A9 \\
\hline 10 & A6 \\
\hline 11 & A3 \\
\hline 12 & A4 \\
\hline 13 & A14 \\
\hline 14 & A1 \\
\hline 15 & A11 \\
\hline
\end{tabular}




\section{CONCLUSION}

Relying on the fact that there are a number of the executive administrations across the nation and that it is not possible to connect all administrations to this network simultaneously and on parallel basis, it has been decided that first according an instruction notified to the central administrations stating that all administrations, general office and all departments across cities and counties as well as affiliated business to the data center should be connected to the corresponding ministry so that after finalization of specialized network connection of the respective administration, the required measures should be taken to connect the data center of the corresponding administration to share center. Thus, all central administrations shall be connected to this network.

As it has been mentioned earlier, considering connection of national central administrations to this network, no priority has been considered for order of connection of corresponding administrations. Thus, in this research, we have made up our mind to prioritize the administrations so that the said administrations shall be connected to this network based on the said prioritization. Consequently, through application of TOPSIS model, fifteen central national administrations have been prioritized based on eight criteria so that in this great national plan we could the order of connection of administrations based on this prioritization.

\section{REFERENCES}

Bennett، John، "Building relationships for technology transfer"، communicatoons of the ACM، 1996، page 35

Martim، Kenney، "Beyond mass production the Japanese systems"، oxford university press، 1993

Cusumano، A.، "How Microsoft buils software"، communication of the ACM،1997 vol 4، page 53

Venkatraman، " Beyond outsourcing L managing IT Resources as a value center Sloan management"، 1997، page 51

Fagin، Ronals، "comparing information without Leaking IT"، Communication of the ACM، 1996، vol 39، page 77

Beig، L.، Eghbali، N.، Ghavamifar، A.، Kharrat، M.، "A Multi-Dimensional Framework for InterAdministrational Collaboration in a Knowledge Network"، Archives of Sciences journal، vol. 66، no.1، 491-508، January 2013

Mahmoudi، M.T.، Taghiyareh، F.، Forouzideh، N.، Lucas، C. " Evolving ANN Structure using Grammar Encoding and Imperialistic Competitive Algorithm"، Journal of Neural Computing \& Applications، Springer 2012

Mahmoudi، M.T.، Beheshti، M.، Badie، K.، Lucas، C. Taghiyareh، F.، " Content-based Image Retrieval using OWA Fuzzy Linking Histogram"، Journal of Intelligent \& Fuzzy Systems، 2013.

Sohrabi. Abolfazl, Pourbijan. Farrokh, Asayesh. Kourosh (2015) Comparison Analysis of Attitude to Change in Knowledge-Based and Non-Knowledge-Based Organization, Bulletin of the Georgian National Academy of Sciences, vol:8, no:2.

Mahmoudi M.T، Taghiyareh`F. Araabi، B.N، " Neuro-Fuzzy Immune Inspired Classifier for Task-Oriented Texts"، Journal of Intelligent \& Fuzzy Systems، 2013 